\section{Potencial herramienta en la personalización del tratamiento oncológico. Casos clínicos}

\author{
CAROLINA IBÁÑEZ C. ${ }^{1}$, MARCELO GARRIDO S. ${ }^{1}$, LIDIA MEDINA ${ }^{1}$, \\ SUMIE KATO ${ }^{2}$, MARÍA LORETO BRAVO ${ }^{3,6}$, PAMELA GONZÁLEZ ${ }^{3}$, \\ BARBARA OLIVA ${ }^{3}$, JAVIER PIZARRO ${ }^{4}$, EVA BUSTAMANTE $^{5}$, \\ JORGE BRAÑES ${ }^{2}$, MAURICIO CUELLO ${ }^{2}$, GARETH I. OWEN ${ }^{3,6}$
}

\section{In vitro cell response to chemotherapeutic agents, to personalize ovarian cancer treatment. Report of two cases}

Our laboratory has implemented an in vitro assay to estimate the response to chemotherapy in ovarian cancer cells pertaining to individual patients. In two selected patients, we determined the correlation between an in vitro assay of cells from suspected ovarian cancer ascites, with the clinical chemotherapy response. Cancer cells isolated from peritoneal fluid with suspected ovarian cancer were tested for cytotoxicity with corresponding chemotherapy regimens. Circulating Ca125 levels and attending physician consultation determined clinical course and response to chemotherapy. The in vitro assay result correlated with Ca125 levels, progression free survival and attending physician evaluation. The assay predicted correctly the failure of two successive chemotherapy regimes in the first patient, while predicting a favorable clinical response in the second subject.

(Rev Med Chile 2013; 141: 669-673).

Key words: Antineoplastic protocols; Ascitic fluid; Ovarian neoplasms.

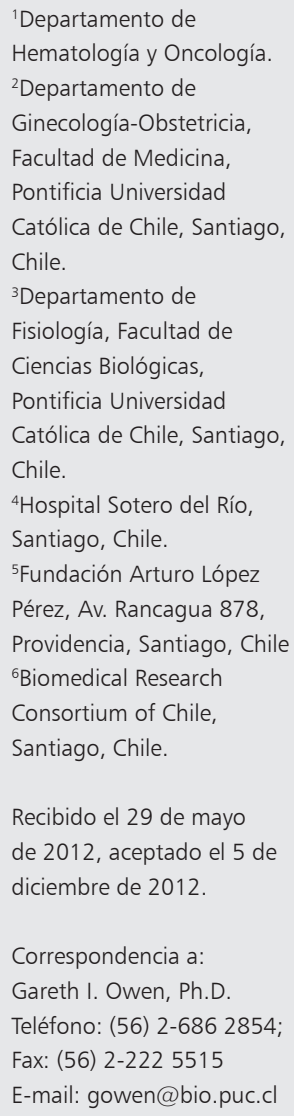

$\mathrm{E}$ n Chile, cáncer de ovario (CO) es la segunda causa de muerte por cáncer ginecológico, con incidencia de 4,4 por 100.000 habitantes ${ }^{1}$. En países desarrollados es de 7/100.000 habitantes $_{1,2}$. De etiología desconocida, se caracteriza por crecimiento lento y $70 \%$ se diagnostica en etapas III o IV $^{3}$. Se disemina en la cavidad abdominal comprometiendo peritoneo. Se confunde frecuentemente con cáncer de origen desconocido peritoneal. La cirugía y quimioterapia (QMT) logran mediana de sobrevida de 30 meses en etapas III-C y menos de 5\% en etapas IV ${ }^{4}$. La QMT obtiene $70-80 \%$ de respuesta y sobrevida sin enfermedad de 12-18 meses, combinando carboplatino-cisplatino y paclitaxel ${ }^{5}$. La respuesta es evaluada clínicamente, con imágenes y nivel plasmático de Ca125, marcador correlacionado con carga tumoral. Para la recurrencia no existe
QMT estándar, las que ofrecen poco más de 25\% de respuesta. Por estos motivos, y por la falta de respuesta inicial de $20-30 \%$ de las pacientes, es imperativo tener nuevas herramientas terapéuticas ${ }^{6}$.

La personalización de la QMT es la situación ideal en oncología 7 . En la década 1980-89 se desarrollaron ensayos con cultivos de células extraídas del tumor para predecir la respuesta a QMT. Se denominó quimiosensibilidad $(\mathrm{QS})^{8}$. La correlación obtenida cuando la QMT no funciona in vitro y la respuesta clínica fue cercana al $80-90 \%$. Pero la correlación entre la respuesta in vitro y la evolución clínica fue $60 \%{ }^{9}$. Por esta razón, se decidió no continuar con estos ensayos ${ }^{10}$.

Ahora, 30 años después, sabemos más de biología tumoral, la naturaleza y características de las células cancerosas, metastáticas, y de las células madre de cáncer ("cancer stem cells"). Todo ello 
nos permite plantear una nueva implementación de este tipo de ensayo. Desde el año 2008, en la Facultad de Ciencias Biológicas y Medicina de la Pontificia Universidad Católica de Chile se realiza el estudio de medicina personalizada en pacientes con cáncer, inicialmente en CO. En este momento se está aplicando esta tecnología en un proyecto de investigación con el fin a largo plazo, de orientar y personalizar los tratamientos ${ }^{7}$.

Postulamos que si el tumor es extraído durante la citorreducción, la QMT posterior estaría dirigida hacia células que escaparon del tumor primario, presentes en la circulación y peritoneo. El modelo óptimo para el análisis de QS y resistencia son células con capacidad de generar focos metastáticos: las contenidas en ascitis. Hemos calculado y experimentado con la concentración y combinaciones de QMT que representan mejor las concentraciones en circulación de pacientes con cáncer. Creemos que esta nueva aproximación va a mantener o mejorar la observación previamente descrita que "si no funciona in vitro, no funciona in vivo". Además esperamos poder entregar una mejor indicación de aquellos tratamientos "que funciona in vitro, funcionará in vivo". Permitiendo mejor respuesta y mayor sobrevida en mujeres con CO. En este artículo presentamos dos ejemplos preliminares de QS en células tumorales aisladas de ascitis y su respectiva correlación clínica.

\section{Material y Método}

\section{Pacientes}

Pacientes mayores de 18 años con sospecha de $\mathrm{CO}$, sometidas a cirugía o paracentesis. Este análisis se realizó en contexto de proyectos de investigación aprobados por el comité de ética de la Pontificia Universidad Católica de Chile, Hospital Sotero del Rio (SSMSO) y la Fundación Arturo López Pérez (SSMO). Las pacientes fueron informadas de los procedimientos aceptando la participación en dichas investigaciones a través de la firma del consentimiento informado.

\section{Metodología}

Muestras de ascitis obtenidas se cultivaron para el ensayo de citotoxicidad. Para este se utilizó MTS (3-(4,5-dimethylthiazol-2-yl)-5-(3 carboxymethoxyphenyl)-2-(4-sulfophenyl)2H-tetrazolium, Cell Titer $96^{\circledR}$ (AQueous One Solution Cell Proliferation Assay, Promega, WI,
EEUU). En este ensayo colorimétrico, la reducción del sustrato se correlaciona con la viabilidad o citotoxicidad en las células vivas ${ }^{11}$. Las QMT utilizadas corresponden a fármacos comerciales utilizados en clínica (Laboratorio farmacéutico Kampar, Santiago, Chile). En el caso de topotecan corresponde al compuesto comercial Hycamtin ${ }^{\circledR}$ (GlaxoSmithKline, Santiago, Chile). Estas drogas se ajustaron a concentraciones descritas en plasma de pacientes que reciben estos tratamientos ${ }^{12,13,14}$. Como control negativo de este ensayo se realizaron tratamientos con la droga ciclofosfamida, que requiere metabolización en el hígado para generar su compuesto activo, siendo inocuo para las células en cultivo. Dentro de este ensayo existe un porcentaje normal de muerte celular que varía entre $10-20 \%$. Con los resultados obtenidos in vitro clasificaremos la respuesta obtenida en 3 grupos: $\mathrm{R}$ (responde a tratamiento, $>50 \%$ ), I (respuesta intermedia $50 \%>\mathrm{X}>30 \%$ ) y $\mathrm{N}$, no responde $(<30 \%)$. Para llevar a cabo las correlaciones con los datos obtenidos in vitro con la respuesta in vivo se analizará la respuesta clínica de las pacientes según criterio del oncólogo tratante y niveles plasmáticos de Ca125, (40U/ml = nivel normal $)$.

\section{Caso 1}

Mujer de 38 años de edad con historia de 3 años de dolores cólicos abdominales, meteorismo y distensión. Al examen físico destacaba abdomen con matidez desplazable. Ca125 de 354 (U/ml), CEA de 4,3 (UI/ml). El resto de los exámenes de laboratorio fueron normales. La tomografía axial computada (TAC) de abdomen mostró una masa anexial derecha que comprometía polo cecal y presencia de carcinomatosis peritoneal. La colonoscopia mostró a nivel del polo cecal una lesión proliferante, exofítica e irregular de $4 \mathrm{~cm}$ de eje mayor. La biopsia concluyó adenoma túbulo velloso con displasia intensa. La endoscopia digestiva alta fue normal. Se realizó paracentesis cuyo block celular confirmó la presencia de carcinoma. Se realizó cultivo de células tumorales de ascitis y estudio de respuesta a QMT según panel utilizado para $\mathrm{CO}$, de acuerdo a lo planificado (Figura 1A). Bajo la sospecha clínica de CO etapa III-C, se inició QMT con cisplatino-paclitaxel sin respuesta. Se repitió colonoscopia cuya biopsia mostró adenoma velloso con displasia intensa. La inmunohistoquímica (IHQ) del block celular 
mostró origen intestinal: CDX2 positivo, Ca125 negativo, citoqueratinas negativo. Se repitió estudio de respuesta a QMT en el ensayo in vitro al nuevo block celular utilizando panel de QMT para cáncer digestivo (Figura 1B). Se cambió la QMT a esquema FOLFOX. Evolucionó con rápido deterioro clínico y falleció. El análisis de QS para panel de CO, mostró citotoxicidad a cisplatino-paclitaxel inferior al 30\% (Figura 1A). El estudio de QS a la progresión para panel de cánceres digestivos ( $\mathrm{Fi}$ gura 1B) mostró citotoxicidad de $20 \%$ al esquema
FOLFOX. Estos resultados muestran correlación entre la respuesta clínica y el resultado de ensayo in vitro tanto para primera como la segunda línea de QMT.

\section{Caso 2}

Mujer de 30 años, que estudio de dolor abdominal con TAC mostró tumor ovárico derecho de $13 \mathrm{~cm}$ y ascitis. Resto del estudio fue normal. Ca125 = $2.526(\mathrm{U} / \mathrm{ml})$. Se realizó cirugía oncoló-

(A)

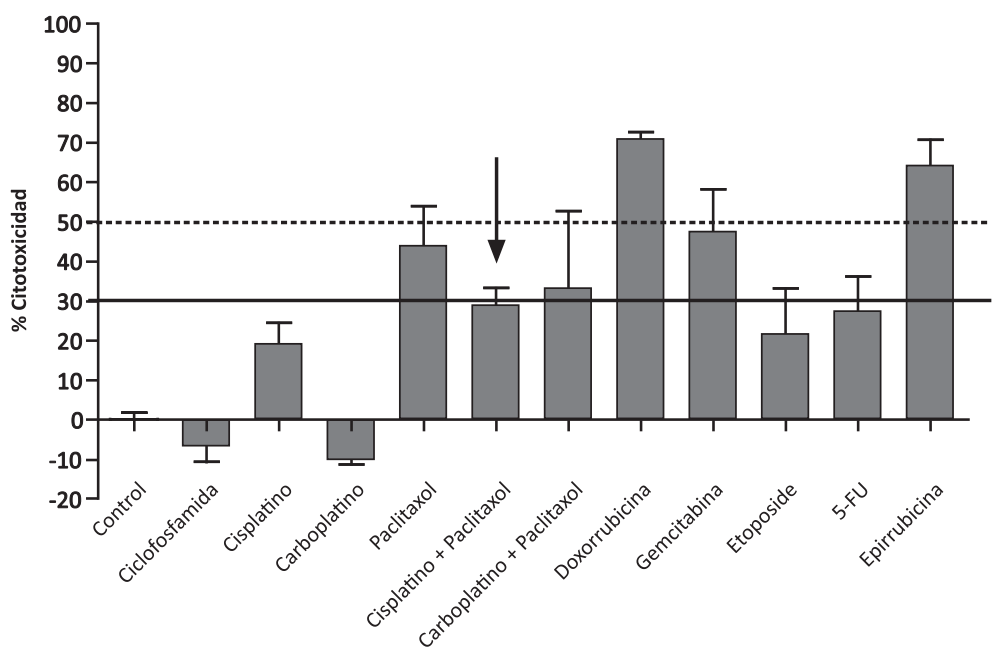

(B)

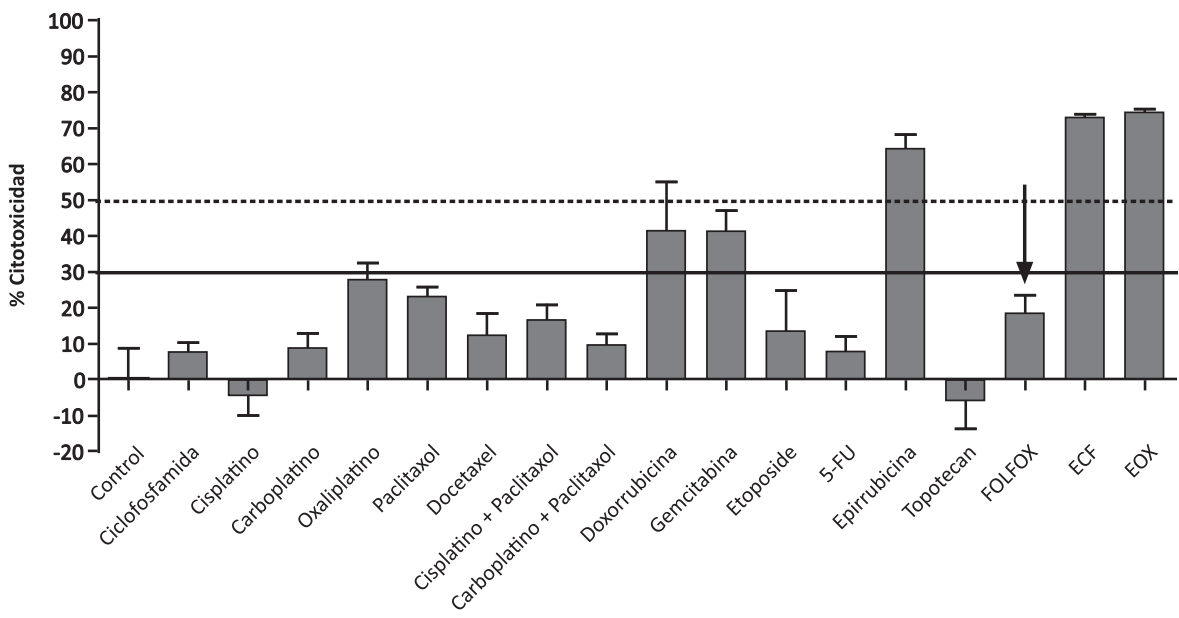

Figura 1. Paciente, (UC052) no presenta respuesta in vitro a los tratamientos administrados, correlacionándose con la respuesta clínica observada. (A) Ensayo de citotoxicidad realizado en cultivo primario de ascitis, donde se analizó distintas quimioterapias para el tratamiento de cáncer de ovario. (B) Ensayo de citotoxicidad realizado en cultivo primario de ascitis proveniente de una recaída de la paciente, donde se analizó tratamientos comúnmente usados en cáncer gástrico/colon. En ambos paneles la flecha indica el tratamiento administrado a la paciente. 


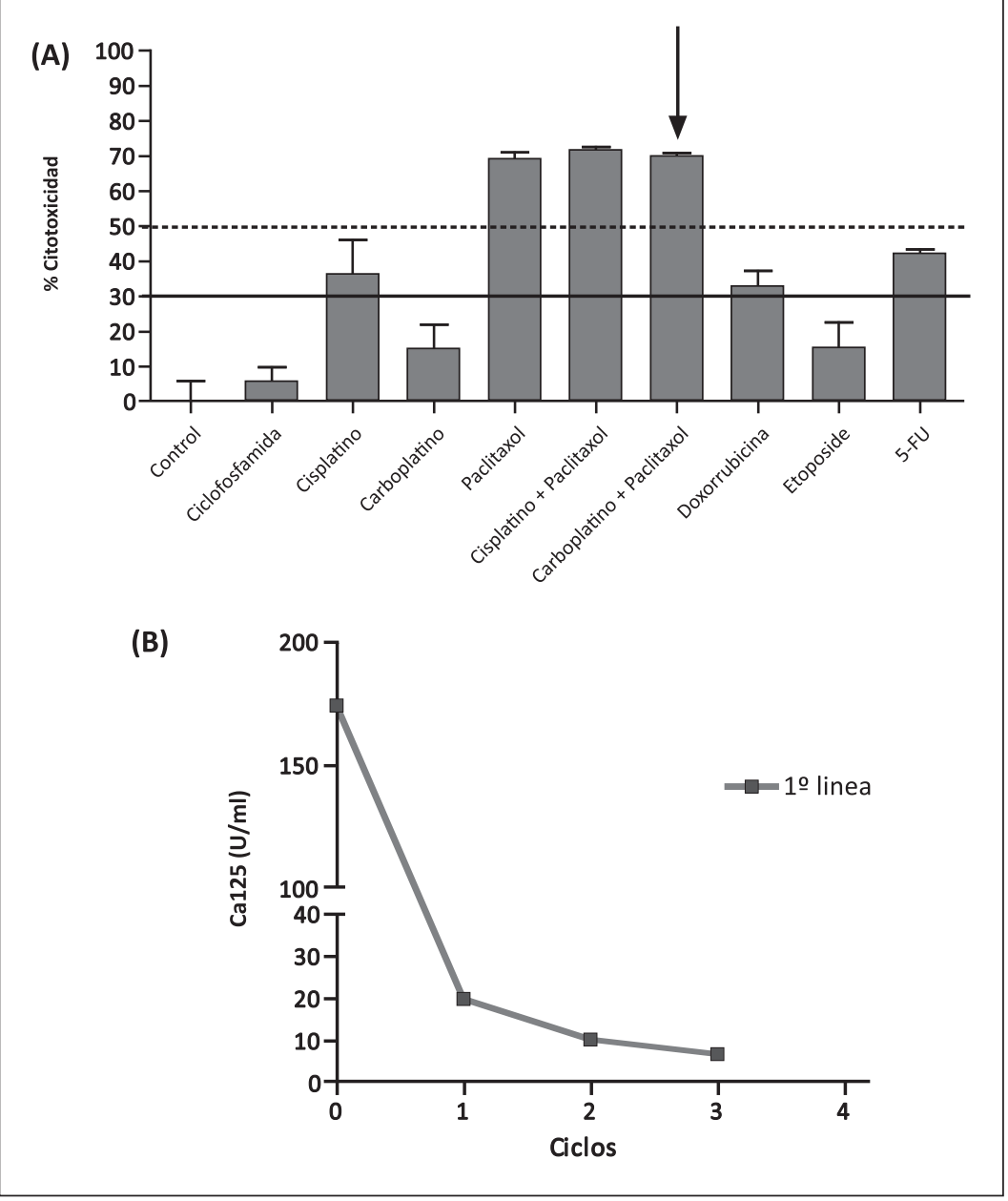

Figura 2. Paciente (UC012) presenta respuesta in vitro a los tratamientos administrados, correlacionándose con una buena respuesta clínica. (A) Ensayo de citotoxicidad realizado en cultivo primario de ascitis, donde se analizó distintas quimioterapias para el tratamiento de cáncer de ovario. (B) Gráfico representando los niveles de reducción del marcador plasmático Ca125 a lo largo de los ciclos de tratamiento. gica. Ca125 post-operatorio $174(\mathrm{U} / \mathrm{ml})$. Biopsia mostró adenocarcinoma endometrioide Grado 3. Etapificada como IC, se tomó muestra de ascitis para block celular y se cultivó para estudio de QS in vitro (Figura $2 \mathrm{~A}$ ). Se administró QMT de carboplatino-paclitaxel, con normalización del Ca125 (Figura 2B). El seguimiento a tres años fue normal. Estudio in vitro mostró nivel de citotoxicidad al esquema carboplatino-paclitaxel mayor al 70\%, correlacionándose con la respuesta clínica.

\section{Discusión}

Cáncer de ovario es cada día más prevalente en países en desarrollo y por este motivo es necesario optimizar los recursos para obtener una mejor respuesta clínica. En CO no existen QMT dirigida.
A pesar de los intentos en la década de 1980-89 mostraron una excelente correlación de las QMT que no funcionan, poca correlación existió entre la respuesta positiva in vitro y la respuesta clínica. Hoy en día, el concepto de la progresión de cáncer cambió. La teoría predominante es la existencia de "células madres del tumor" o "células iniciadoras de metástasis" que escapan del tumor primario y generan micrometástasis. Estas células, tienen respuesta a QMT diferente del tumor primario, y corresponderían por lo tanto, al verdadero blanco de los tratamientos. Por ende, en este reporte utilizamos ascitis de dos pacientes con sospecha de CO. Las células aisladas, teóricamente, corresponden a las que se escaparon del tumor primario y presentan características similares a las células iniciadoras de metástasis. 
Estos dos resultados obtenidos nos sugieren de manera preliminar una posibilidad exploratoria de fácil acceso, que en conjunto con otros indicadores, pudieran orientar futuras herramientas para elección del tratamiento. Este proyecto actualmente está evaluando este ensayo con un mayor número de pacientes de distinto grado tumoral y pronóstico. La meta a largo plazo es poner en marcha los conocimientos adquiridos y permitir que este proyecto se transforme en un ensayo clínico para entregar más información al oncólogo. La entrega de un patrón de respuesta quimioterapéutica para cada paciente seria una herramienta interesantemente útil a explorar en futuros estudios oncológicos.

Agradecimientos: Las pacientes que voluntariamente participaron en este proyecto y el personal médico y de apoyo de los hospitales participantes. The Biomedical Research Consortium of Chile CTU06 (GO). El Fondo Nacional de Desarrollo Científico y Tecnológico Gobierno de Chile FONDECYT 1100870 (GO), 1080163 (MAC) 3120003 (MLB).

\section{Referencias}

1. Donoso, E. Cambio del perfil epidemiológico y demográfico determina un mayor riesgo de cáncer ginecológico en la mujer chilena. Rev Chil Obstet Ginecol 2012; 77 (4): 247-8.

2. Sieguel R, Naishadham D, Jemal A. Cancer Statistics. CA Cancer J Clin 2012; 62: 10-29.

3. Goff B, Mandel L, Muntz H, Melancon C. Ovarian Carcinoma Diagnosisresults of a National Ovarian Cancer Survey. Cancer 2000; 15: 2068-75.

4. Pignata S, Cannella L, Leopardo D, Pisano C, Bruni G, Facchini G. Chemotherapy in epithelial ovarian cancer. Cancer Letters 2011; 303: 73-83.
5. Mutch DG. Surgical management of ovarian cancer. Semin Oncol 2002; 29 (1 Suppl 1): 3-8.

6. Jelovac D, Armstrong D, Recent Progress in the Diagnosis and Treatment of ovarian Cancer. CA Cancer J Clin 2011; 61: 183-203.

7. The Cancer Genome Atlas Research Network. Integrated genomic analyses of ovarian carcinoma. Nature, 2011; 474: 609-15.

8. Cree IA, Kurbacher CM, Lamont A, Hindley AC, Love S; TCA Ovarian Cancer Trial Group. A prospective randomized controlled trial of tumour chemosensitivity assay directed chemotherapy versus physician's choice in patients with recurrent platinum-resistant ovarian cancer. Anticancer Drugs 2007; 18 (9): 1093-101.

9. Shink JC, Copeland LJ. Point: chemosensitivity assays have a role in the management of recurrent ovarian cancer. J Natl Compr Cancer Netw 2011; 1: 115-20.

10. Herzog TJ, Krivak TC, Fader AN, Coleman RL. Chemosensitivity tetsing with ChemoxFx and overall survival in primary ovarían Cancer. Am J Obstet Gynecol 2010; 203: 68. e1-6.

11. Sadarangani A, Kato S, Espinoza N, Lange S, Llados C, Espinosa $\mathrm{M}$, et al., TRAIL mediates apoptosis in cancerous but not normal primary cultured cells of the human reproductive tract. Apoptosis. 2007; 12 (1): 73 85.

12. Hunz M, Jetter A, Warm M, Pantke E, Tuscher M, Hempel G, et al. Plasma and tissue pharmacokinetics of epirubicin and Paclitaxel in patients receiving neoadjuvant chemotherapy for locally advanced primary breast cancer. Clin Pharmacol Ther. 2007; 81 (5): 659-68.

13. Nagai N, Kinoshita M, Ogata H, Tsujino D, Wada Y, Someya K, et al. Relationship between pharmacokinetics of unchanged cisplatin and nephrotoxicity after intravenous infusions of cisplatin to cancer patients. Cancer Chemother Pharmacol. 1996; 39 (1-2): 131-7.

14. Celio LA, DiGregorio GJ, Ruch E, Pace J, Piraino AJ. Doxorubicin and 5-fluorouracil plasma concentrations and detectability in parotid saliva. Eur J Clin Pharmacol 1983; 24 (2): 261-6. 